DOI: $10.2478 /$ ace-2013-0006

\title{
ON ADJUSTING THE LOAD BEARING CAPACITY OF DECISIVE MEMBERS TO RELIABILITY CLASSES OF STATICALLY DETERMINATE COMPLEX STRUCTURES
}

\section{Z. KOWAL ${ }^{1}$}

\begin{abstract}
The paper provides a solution to the problem of dimensioning decisive bars on the basis of the conditions of meeting the recommended reliability classes [9] of statically determinate structures composed of $\mathrm{n}$ members. A theorem was formulated: if a statically determinate structure composed of $\mathrm{n}$ decisive members is to attain the reliability greater than, or equal to, the recommended reliability $p=1-q$, it is necessary and sufficient that the damage frequency sum $q_{i}$ of decisive members is smaller than the admissible damage frequency $\mathrm{q}$ of the structure: $\sum \mathrm{q}_{\mathrm{i}}<\mathrm{q}$. On the basis of this theorem, $s$ coefficients that recommend increase of the load bearing capacity of the decisive bars in a statically determinate structure constructed in order to meet the recommended class [9] of the structure reliability, are estimated and presented in a tabular form.
\end{abstract}

Key words: reliability, complex structures, quantiles of member load bearing capacity

\section{INTRODUCTION}

The degradation of the load bearing capacity and reliability of statically determinate structures composed of members calculated in accordance with the code, including the EU code [9], is a known problem. The degradation of the load bearing capacity in time is caused by steel structure deterioration due to: 1) aging of material, 2) corrosion that occurs, in particular, in industrial buildings (e.g. industrial stacks, pipelines, etc.), 3) corrosion and steel abrasion in hydro-engineering, 3) member fatigue in machine engineering, 4) documentation errors. In the paper, the impact of the load bearing capacity of joints, and that one of the number of decisive members, on reduced load bearing capacity of statically determinate structures was taken into account. Those impacts were accounted for while estimating the coefficients that increase the load bearing capacity of decisive bars, thus restoring the recommended reliability class [9] of complex structures. When in some construction practice, joints with load bearing capacity lower than the load bearing capacity of bars jointed at nodes are used, a considerable number of decisive members results in reduced load bearing capacity and reliability of bar structures, and may lead to failure and collapse of complex structures. In B2 Table of the code

\footnotetext{
1 Kielce University of Technology, Al. Tysiąclecia P.P. 7, 25-314 Kielce, Poland.
} 
[9], three structure reliability classes with the recommended minimum reliability index after 50 years of service are differentiated. Those include: $\mathbf{R C 1}$ class with the reliability index $\boldsymbol{\beta}_{0}=3.3, \mathrm{RC} 2$ class $-\boldsymbol{\beta}_{\mathbf{0}}=\mathbf{3 . 8}$, and $\mathrm{RC} 3$ class $-\boldsymbol{\beta}_{\mathbf{0}}=\mathbf{4 . 3}$. Minimum reliability indexes $\beta$ refer to the ultimate strength limit states. Below B2 Table, an annotation was made: generally, it is thought that designing a structure in accordance with EN 1990 [9] with partial indexes given in the Appendix A1 and EN 1991 to EN 1999 [10], yields a structure with the reliability index $\beta>\mathbf{3 . 8}$ for the $\mathbf{5 0}$-year reference period.

The information quoted in the code [9] is based on practical experience collected from observations of the structure service life, without differentiation made between mechanical properties of structures constructed of different simple and complex decisive members. The term "decisive members" is used in reference to minimum critical sets of members, the destruction of which activates the kinematically admissible failure mechanism (KAFM) of the structure, resulting in the collapse of the structure, or of its part. In the paper, symbol $\mathbf{t}$ denotes the index of structure reliability. It replaces the notation used in the code, i.e. $\boldsymbol{\beta}$. The aim is to differentiate the impact of the number of decisive members, including the load bearing capacity of bars and their connections with nodes, on a decrease in load bearing capacity and reliability of structures composed of many members, from the impact resulting from wear. The reliability index $\boldsymbol{\beta}_{\mathbf{2}}=\mathbf{t}_{\mathbf{2}}=\mathbf{3 . 8}$ recommended for RC2 class can be adopted as a reliability index for simple and complex decisive members of steel structures, designed of non-aging structural steels, protected against corrosion, in accordance with the presently binding code. The reliability index mentioned above is recommended by the code [9] and it has proved effective in construction practices. On the basis of historical experience collected in construction, it can be assumed that decisive members of steel structures, dimensioned in accordance with the presently binding code, indicate the reliability index $t_{2}=3.8$, which is congruent with the content of the code [9]. The structural reliability $\mathrm{p}$ of statically determinate $\mathrm{RC} 2$ class bar structures composed of decisive members having the reliability RC2, is estimated from the formula (1.1) [5]:

$$
\mathbf{p}=\prod \operatorname{Pr}\left\{\mathbf{N}_{\mathbf{i}}>\mathbf{S}_{\mathbf{i}}\right\}>\mathbf{p}(\mathbf{t}=\mathbf{3 . 8}), \text { for } \mathbf{i} \text { from } \mathbf{1} \text { to } \mathbf{n}
$$

where: $\mathrm{N}_{\mathrm{i}}$ - random load bearing capacity of bars, $\mathrm{S}_{\mathrm{i}}-$ random forces in bars, resulting from the structure load.

From the formula above, it is obvious that the more decisive members are found in a statically determinate structure, the lower is its reliability. Thus, it is important to develop a method of restoring the recommended class reliability, especially for large complex structures, by means of estimating $\mathbf{s}$ coefficients, which appropriately increase [9] the reliability of decisive members. Fig 1 . shows an example of statically determinate structure, composed of decisive members in the form of bars, and their connections with the nodes.

Integrated decisive member 3 , shown in Fig. $1 \mathrm{~b}$, is a specific complex member, in which three potentially decisive members occur: a bar (3) and two joints $(1 ; 2)$. In 
order to regard bar 3, and joints 1 and 2, as a single integrated decisive member, it is necessary to construct joints 1 and 2 in accordance with paper [7], i.e. with increased load bearing capacity when compared with bar 3 .

a)
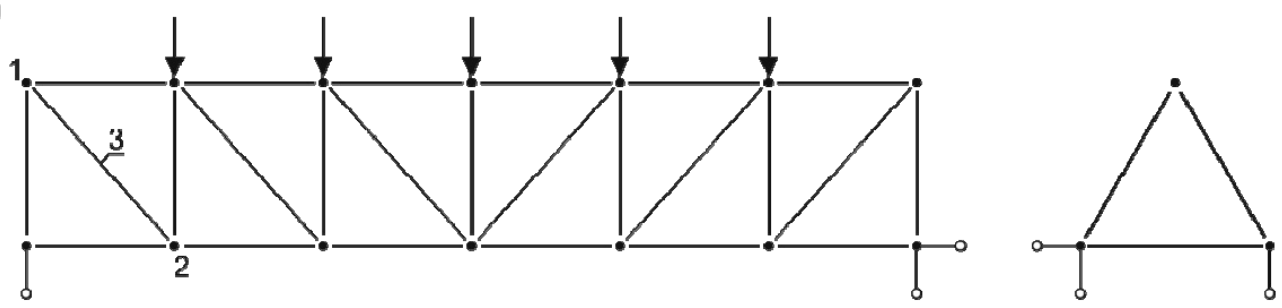

b)

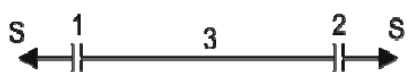

Fig. 1. Examples: a) statically determinate complex structure,

b) complex decisive member

2. AdJUSTING THE LOAD BEARING CAPACITY OF DECISIVE MEMBERS TO RECOMMENDED

RC2 ClASS RELIABILITY OF STATICALLY DETERMINATE COMPLEX STRUCTURES

Estimating the postulated increase in the reliability $\mathbf{p}\left(\mathbf{t}_{\mathbf{j}}\right)$ of decisive members in a statically determinate complex structure, which satisfy the equation (2.1), is meant to meet the recommended reliability class $\mathbf{p}\left(\mathbf{t}_{\mathbf{2}}\right)$ of the whole complex structure:

$$
\prod \mathbf{p}\left(\mathbf{t}_{\mathbf{i}}\right)>=\mathbf{p}\left(\mathbf{t}_{2}\right)
$$

Each decisive member, dimensioned in accordance with the codes, has the expected reliability at the level of recommended reliability $\mathbf{p}\left(\mathbf{t}_{\mathbf{2}}=\mathbf{3 . 8}\right)$ of the structure, which is forecasted to be found at the end of the structure service life [9]. In order to meet the recommended reliability $\mathbf{p}\left(\mathbf{t}_{\mathbf{2}}=\mathbf{3 . 8}\right)$ of the whole statically determinate complex structure, it is necessary to increase the reliability of decisive members $\mathbf{p}\left(\mathbf{t}_{\mathbf{i}}\right)$ so that the equation (2.2) is satisfied:

$$
\mathbf{p}\left(\mathbf{t}_{2}\right)=\mathbf{1}-\mathbf{q}\left(\mathbf{t}_{2}\right)=\prod \mathbf{p}\left(\mathbf{t}_{\mathbf{i}}\right)=\mathbf{1}-\sum \mathbf{q}\left(\mathbf{t}_{\mathbf{i}}\right)
$$

where: $\mathbf{p}\left(\mathbf{t}_{\mathbf{2}}=\mathbf{3 . 8}\right)$ - the structure reliability recommended in $\mathbf{R C 2}$ class, $\mathbf{q}\left(\mathbf{t}_{\mathbf{2}}\right)=\mathbf{1}-\mathbf{p}\left(\mathbf{t}_{\mathbf{2}}=\mathbf{3 . 8}\right)$ - admissible damage frequency of the structure, $\mathbf{q}\left(\mathbf{t}_{\mathbf{i}}\right)$ - damage frequency of decisive members, which makes it possible to meet the recommended reliability class of a complex structure. 
In a statically determinate complex structure, $\mathrm{n}$ decisive members are found. In order to meet the recommended reliability level $\mathbf{p}\left(\mathbf{t}_{2}\right)$ of a complex structure, it is necessary to restrict the maximum damage frequency $\mathbf{q}\left(\mathbf{t}_{\mathbf{i}}\right)$ of decisive members to the one determined from the equation (2.3):

$$
\mathbf{q}\left(\mathbf{t}_{\mathbf{i}}\right)=\mathbf{q}\left(\mathbf{t}_{2}\right) / \mathbf{n}
$$

The resultant damage frequency $\mathbf{q}\left(\mathbf{t}_{\mathbf{i}}\right)$ of members can be globally checked from the inequality (2.4):

$$
\sum \mathbf{q}\left(\mathbf{t}_{\mathbf{i}}\right)<\mathbf{q}\left(\mathbf{t}_{\mathbf{2}}\right)
$$

The reliability of members in statically determinate complex structure should satisfy the theorem 1: if a statically determinate structure composed of $\mathbf{n}$ decisive members is to attain the reliability $p$ greater than, or equal to, the recommended class of structure reliability $p=1-q$, it is necessary and sufficient that the damage frequency sum $q_{i}$ of decisive members is smaller than the damage frequency $q$ of the complex structure $\sum \mathbf{q}_{\mathbf{i}}<\mathbf{q}$.

For RC2 class structures, the recommended reliability of decisive members can be estimated from the formula (2.5):

$$
p\left(t_{i}\right)=1-q\left(t_{i}\right)>1-q\left(t_{2}\right) / n
$$

On the basis of $\mathbf{p}\left(\mathbf{t}_{\mathbf{i}}\right)$, an increased reliability index $\mathbf{t}_{\mathbf{i}}$ for decisive members can be read from the load bearing capacity distribution tables [11]. The aim of estimating an increase in the reliability index $t_{i}$ of decisive members in a complex structure composed of $\mathrm{n}$ decisive members is to meet the recommended reliability index of the structure which belongs to one of RC1, RC2, RC3 complex structure reliability classes [9]. An increase of the load bearing capacity of decisive members dimensioned in accordance with the codes [10] should correspond to an increased quantiles (2.6) with the reliability index $t_{i}$ of decisive members determined from the equation (2.5):

$$
\mathbf{N}_{\mathrm{izk}}=\mathbf{E}\left(\mathbf{N}_{\mathrm{iz}}\right)\left[1-\mathbf{t}_{\mathrm{i}} \mathbf{V}_{\mathrm{i}}\right],
$$

where: $\mathbf{E}\left(\mathbf{N}_{\mathbf{i z}}\right)$ - expected load bearing capacity of the i-th strengthened member, $\mathbf{t}_{\mathbf{i}}-$ reliability index of strengthened members, $\mathbf{v}_{\mathrm{i}}=\mathbf{D}\left(\mathbf{N}_{\mathrm{iz}}\right): \mathbf{E}\left(\mathbf{N}_{\mathrm{iz}}\right)=\mathbf{D}\left(\mathbf{N}_{\mathrm{i}}\right): \mathbf{E}\left(\mathbf{N}_{\mathrm{i}}\right)-$ coefficient of variation in the load bearing capacity of members, $\mathrm{D}\left(\mathrm{N}_{\mathrm{iz}}\right)$ - standard deviation of the i-th strengthened member.

Coefficients $\mathbf{s}$ of load bearing capacity of decisive members are determined from the equation (2.7) of quantiles of decisive members having the reliability stated by the code (2.1) and the reliability postulated (2.6) for a complex structure:

$$
E\left(N_{i z}\right)\left[1-t_{i} v_{i}\right]=E\left(N_{i}\right)\left[1-t_{2} v_{i}\right] .
$$


Coefficients $\mathbf{s}$ which requires an increase in the load bearing capacity of decisive members, are estimated on the basis of equation (2.7), from the formula (2.8):

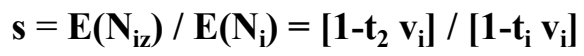

Increased quantiles of the load bearing capacity of decisive members result from the need to increase expected load bearing capacities $\mathbf{E}\left(\mathbf{N}_{\mathbf{i z}}\right)$. Increasing the expected value, by definition, does not change the coefficient of variation of the load bearing capacity of decisive members, fabricated while following the same technology. In decisive members, different coefficients of the load bearing capacity variation $\mathbf{v}_{\mathbf{i}}$ of members can occur, which does not change the manner of estimation. In numerical examples, the following are taken into account: the most frequently occurring, identical coefficients of the load bearing capacity - variation $v_{i}$ of members, and equal indexes $t=t_{i}$ of the reliability of decisive members, depending on the number $\mathbf{n}$ of all decisive members found in the complex structure under consideration. Coefficients $\mathbf{s}$ of the load bearing capacity of members estimated from the formula (2.8) should be applied to increase the load bearing capacity of decisive members, which have already been estimated in accordance with respective codes.

The equalisation of reliabilities of statically determinate structures composed of many decisive members makes it possible to adjust reliabilities of decisive members to recommended reliability class of statically determinate complex structures by means of employing historical experience in structure dimensioning, which is contained in academic procedures and construction codes.

\section{Numerical adJustment of COMPUTATIONAL LOAD BEARING CAPACITIES OF DECISIVE ELEMENTS TO THE RELIABILITY OF RC2 CLASS COMPLEX STRUCTURES}

The manner of adjusting the load bearing capacity and reliability of decisive members to commonly accepted structure RC2 class with the reliability index $\mathbf{t}_{\mathbf{2}}=\mathbf{3 . 8}$ is shown with an example of a bar structure composed of 10 members.

Example 1. Recommended [9] in general construction, structure RC2 class reliability with the reliability index $\mathbf{t}_{2}=\mathbf{3 . 8}$ amounts to $[10]$ : $\mathbf{p}\left(\mathbf{t}_{\mathbf{2}}=\mathbf{3 . 8}\right)=\mathbf{0 . 9 9 9 9 2 7 6 5 2}$. The corresponding structure damage frequency is: $\mathbf{q}\left(\mathbf{t}_{2}=\mathbf{3 . 8}\right)=\mathbf{1}-\mathbf{p}\left(\mathbf{t}_{2}=\mathbf{3 . 8}\right)=\mathbf{0 . 0 0 0 0 7 2 3 4 8}$. The admissible damage frequency of the decisive members of the structure composed of $\mathbf{1 0}$ members is: $\mathbf{q}(\mathbf{t})=\mathbf{q}\left(\mathbf{t}_{2}\right) / \mathbf{1 0}=\mathbf{0 . 0 0 0 0 0 7 2 3 4 8}$, the reliability: $\mathbf{p}\left(\mathbf{t}_{\mathbf{i}}\right)=\mathbf{1}-\mathbf{q}\left(\mathbf{t}_{\mathbf{2}}\right)$ $/ \mathbf{1 0}=\mathbf{0 . 9 9 9 9 9 2 7 6 5 2}$. The recommended reliability index of a member is [11]: $\mathbf{t}=\mathbf{4 . 3 3 6 6 5}$. The coefficient $\mathbf{s}$ for $\mathbf{v}_{\mathbf{i}}=\mathbf{0 . 1}$ computed from the formula (2.8) amounts to: $s=[1-3.8 \times 0.1) /[1-4.33665 \times 0.1]=0.62 / 0.56634=1.0948$. Increasing, in accordance with Example 1, the load bearing capacity of bars in a statically determinate structure of 10 decisive bars having the reliability index $\mathbf{t}_{\mathbf{s}} \mathbf{= 4 . 3 3 6 6 5}$, restores the recommended reliability of complex structure according to the estimation. The 
reliability of the structure composed of $\mathbf{1 0}$ bars is as follows: $\mathbf{p}=\prod \mathbf{p}_{\mathbf{s i}}=\mathbf{1}-\mathbf{1 0} \mathbf{q}_{\mathrm{s}}=$ $1-\mathbf{0 . 0 0 0 0 7 2 3 4 8}=\mathbf{0 . 9 9 9 9 2 7 6 5 2}$. From normal distribution table $[11]$, we have: $\mathbf{t}=\mathbf{3 . 8}$. The structure demonstrates the reliability recommended by the code [9].

On the basis of Example 1, coefficients s recommending an increase in the load bearing capacity of the decisive members of statically determinate trusses composed of $\mathbf{n}=10 ; 50 ; 100 ; 200 ; 500 ; 800 ;$ and 1000 decisive members, with the variation coefficient of load bearing capacity $\mathbf{v}=\mathbf{0 . 0 6} ; \mathbf{0 . 0 8} ; \mathbf{0 . 1} ; \mathbf{0 . 1 2}$, are estimated and put into tables.

Table 1

Coefficients $\mathbf{s}$ increasing the load bearing capacity of decisive members of RC2 class structure $\left(\mathbf{t}_{\mathbf{2}}=\mathbf{3 . 8}\right)$

\begin{tabular}{|c|c|c|c|c|c|c|c|c|}
\hline & $\mathbf{n}$ & $\mathbf{q}$ & $\mathbf{p}=\mathbf{1}-\mathbf{q}$ & $\mathbf{t}_{\mathbf{i}}=$ & $\mathbf{v}=\mathbf{0 . 0 6}$ & $\mathbf{v}=\mathbf{0 . 0 8}$ & $\mathbf{v}=\mathbf{0 . 1}$ & $\mathbf{v}=\mathbf{0 . 1 2}$ \\
\hline item & 1 & 2 & 3 & 4 & 5 & 6 & 7 & 8 \\
\hline 1 & 1 & 0.000072348 & 0.999927652 & 3.8 & 1 & 1 & 1 & 1 \\
\hline 2 & 10 & 0.0000072348 & 0.9999927652 & 4.3367 & $\mathbf{1 . 0 4 2 6}$ & $\mathbf{1 . 0 6 7 2}$ & $\mathbf{1 . 1 0 2 6}$ & $\mathbf{1 . 1 5 8 2}$ \\
\hline 3 & 100 & 0.00000072348 & 0.9999992765 & 4.8185 & $\mathbf{1 . 0 8 6 0}$ & $\mathbf{1 . 1 3 4 4}$ & $\mathbf{1 . 2 0 5 2}$ & $\mathbf{1 . 3 1 6 4}$ \\
\hline 4 & 200 & 0.0000003618 & 0.9999996382 & 4.9550 & $\mathbf{1 . 0 9 8 1}$ & $\mathbf{1 . 1 5 4 6}$ & $\mathbf{1 . 2 3 6 1}$ & $\mathbf{1 . 3 6 4 1}$ \\
\hline 5 & 500 & 0.0000001447 & 0.9999998553 & 5.1303 & $\mathbf{1 . 1 1 5 1}$ & $\mathbf{1 . 1 8 1 3}$ & $\mathbf{1 . 2 7 6 9}$ & $\mathbf{1 . 4 2 7 0}$ \\
\hline 6 & 1000 & 0.00000007235 & 0.99999992765 & 5.2592 & $\mathbf{1 . 1 2 7 9}$ & $\mathbf{1 . 2 0 1 5}$ & $\mathbf{1 . 3 0 7 8}$ & $\mathbf{1 . 4 7 4 7}$ \\
\hline
\end{tabular}

Table 1 comprises in columns: 1 - number of decisive members in a structure, 2 - damage frequency of decisive members, 3 - recommended reliability of members, 4 - recommended reliability index of members. Columns 5, 6, 7, 8 of Table 1 provide coefficients $\mathbf{s}$, estimated from formula (2.8) as a function of n number of members, which recommend an increase in the load bearing capacity of decisive members. Validation of statically determinate structure composed of $\mathbf{1 0 0 0}$ decisive bars, each having the reliability index $t_{s}=\mathbf{5 . 2 5 9 2}$, reliability $p_{s}=\mathbf{0 . 9 9 9 9 9 9 9 2 7 6 5 2}$, damage frequency $\mathbf{q}_{\mathbf{s}}=\mathbf{1}-\mathbf{p}_{\mathbf{s}}=\mathbf{= 0 . 0 0 0 0 0 0 7 2 3 4 8}$, indicates that the reliability of such structure is: $\mathbf{p}=\prod p_{\mathrm{si}}=\mathbf{1}-\mathbf{1 0 0 0} \mathbf{q}_{\mathrm{s}}=\mathbf{1}-\mathbf{0 . 0 0 0 0 7 2 3 4 8}=\mathbf{0 . 9 9 9 9 2 7 6 5 2}$. The reliability index is: $\mathbf{t}=\mathbf{3 . 8}$, which is congruent with the code recommendations [9].

On the basis of Table 1, graphs shown in Fig. 2 are plotted in order to estimate coefficients $\mathbf{s}$ by using the logarithmic abscissa scale $\lg _{\mathbf{1 0}}(\mathbf{n})$ of the $\mathbf{n}$ number of members. The practical reason for employing the logarithmic abscissa scale is the possibility of accurate interpolation and extrapolation of coefficients $\mathbf{s}$, because graphs of coefficients $\mathbf{s}$ are rectilinear when the logarithmic abscissa scale $\lg _{\mathbf{1 0}}(\mathbf{n})$ is used. This mode is shown in Example 2.

Example 2. Estimation of coefficients $\mathbf{s}$ for $\mathrm{v}_{\mathrm{i}}=0.09$ by controlling the load bearing capacity of the members with the logarithmic abscissa scale. RC-2 class truss [9] 
consists of $\mathbf{n}=\mathbf{1 0 0 0}$ decisive members. The reliability index $\mathrm{t}$ [11] of members amounts to: $\mathrm{t}=\mathbf{5 . 2 5 9 2}, \mathbf{s}$ coefficient $=\left[\mathbf{1}-\mathbf{t}_{\mathbf{2}} \mathbf{v}_{\mathbf{i}}\right] /\left[\mathbf{1 - t} \mathbf{v}_{\mathbf{i}}\right]=(1-3.8 \times 0.09) /(1-\mathbf{5 . 2 5 9 2} \times 0.09)=0.658$ / $0.526672=\mathbf{1 . 2 4 9 4}$.

Table 2 presents coefficients $\mathbf{s}$ for $\mathrm{v}=0.09$ interpolated in accordance with Example 2 .

Table 2

Coefficients $\mathbf{s}$ as a function of $\mathrm{n}$, obtained from interpolation for $\mathrm{RC} 2$ class structure

\begin{tabular}{|c|c|c|c|c|c|c|c|}
\hline & $\mathrm{n}=10$ & 50 & 100 & 200 & 500 & 800 & $\mathbf{1 0 0 0}$ \\
\hline $\lg _{10}(\mathrm{n})$ & 1 & 1.6990 & 2 & 2.3010 & 2.6990 & 2.9031 & 3 \\
\hline $\mathrm{v}=0.09$ & $\mathbf{1 . 0 8 3 1}$ & $\mathbf{1 . 1 4 1 2}$ & $\mathbf{1 . 1 6 6 2}$ & $\mathbf{1 . 1 9 1 3}$ & $\mathbf{1 . 2 2 4 3}$ & $\mathbf{1 . 2 4 1 3}$ & $\mathbf{1 . 2 4 9 4}$ \\
\hline
\end{tabular}

With the use of the logarithmic abscissa scale, the graphs in Fig. 2 for $\mathrm{n}=10000$ are completed. That is done on the basis of extrapolation, owing to the fact that the straight line graphs go through the centre of the co-ordinate system $(1 ; 1)$. In Fig. 2, rectilinear $\mathbf{s}$ dependence on $\mathbf{n}$ is shown, which was obtained with the help of the logarithmic abscissa scale $\lg _{10}(\mathrm{n})$.

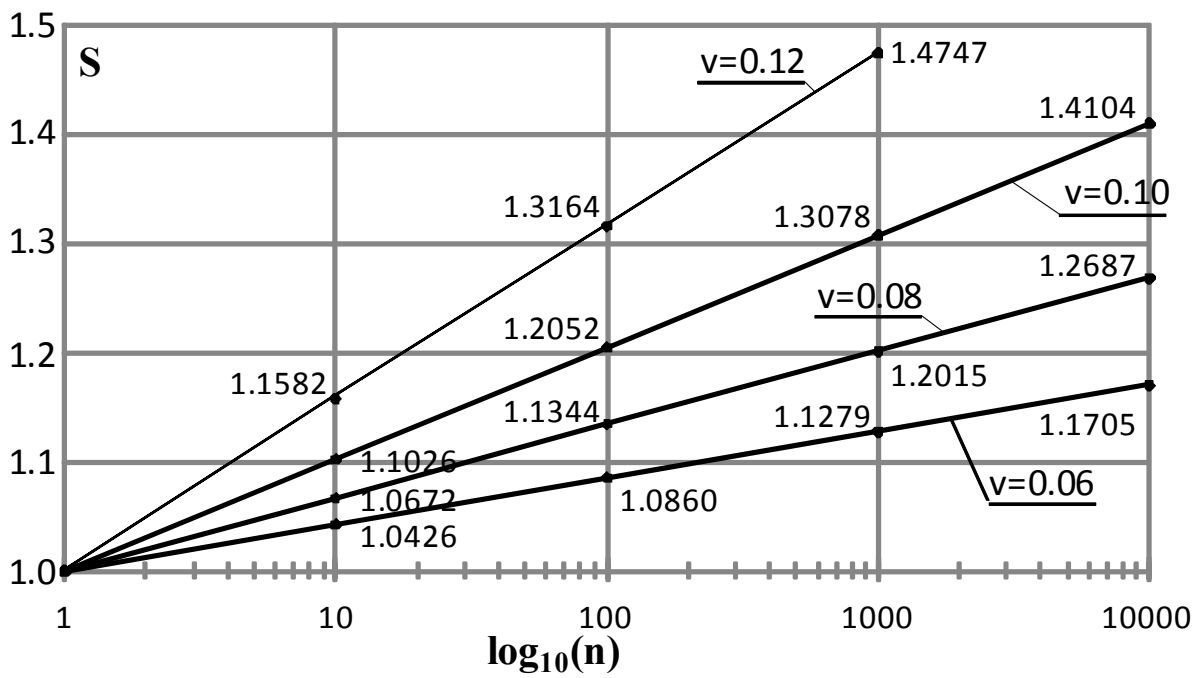

Fig. 2. Coefficients $\mathbf{s}$ increasing the load bearing capacity of decisive elements of RC 2 class structure $\left(t_{2}=3.8\right)$.

While designing statically determinate $\mathrm{RC} 2$ class structures, in accordance with the formula (2.8), it is also necessary to increase, with the help of coefficients $\mathbf{s}$, the load bearing capacity of decisive members dimensioned in accordance with detailed codes. 
4. Numerical adJUSTMENT OF COMPUTATIONAL LOAD BEARING CAPACITIES OF DECISIVE ELEMENTS TO THE RELIABILITY OF RC3 CLASS COMPLEX STRUCTURES

The recommended reliability index for $\mathbf{R C} 3$ class structure [9] is: $\mathbf{t}_{\mathbf{3}}=\boldsymbol{\beta}=\mathbf{4 . 3}$. It should be taken into account that the more decisive members are found in a structure, the more serious are the consequences of a failure or a collapse. A high number of decisive members is used in large structural systems, e.g. bar structures or tendon structures of large volume halls, in tall frame buildings, and in many other structures. Let us consider high-profile statically determinate RC3 class structures and estimate coefficients $\mathbf{s}$ equalizing the structure reliability in the logarithmic abscissa scale for the $\mathbf{n}$ number of decisive members. It is assumed that, as expected, each decisive member, dimensioned in accordance with the code, has the expected initial reliability level, which is congruent with the structure reliability stated in the code $\mathbf{p}\left(\mathbf{t}_{\mathbf{2}}=\mathbf{3 . 8}\right)$. In order to obtain increased reliability $\mathbf{p}\left(\mathbf{t}_{3}=\mathbf{4 . 3}\right)=\mathbf{0 . 9 9 9 9 9 1 4 6 0 0 9 4 5 2 4}$ (related to the damage frequency $\mathbf{q}=\mathbf{1}-\mathbf{p}\left(\mathbf{t}_{\mathbf{3}}=\mathbf{4 . 3}\right)=\mathbf{0 . 0 0 0 0 0 8 5 3 9 9 0 5 4 7 6 )}$ recommended for the whole statically determinate structure of $\mathbf{R C 3}$ class, it is necessary to increase the reliability of the decisive members so that the equation (3.1) is satisfied:

$$
\mathbf{p}\left(\mathbf{t}_{3}\right)=\mathbf{1}-\mathbf{q}\left(\mathbf{t}_{3}\right)=\prod \mathbf{p}_{\mathbf{i}}(\mathbf{t})=\left[1-\sum \mathbf{q}_{\mathbf{i}}(\mathbf{t})\right],
$$

where: $p\left(t_{\mathbf{3}}=\mathbf{4 , 3}\right)$ - structure reliability recommended for $\mathbf{R C 3}, \mathbf{q}\left(\mathbf{t}_{\mathbf{3}}\right)=\mathbf{1}-\mathbf{p}\left(\mathbf{t}_{\mathbf{3}}\right)$ - admissible damage frequency of the structure, $\mathbf{q}\left(\mathbf{t}_{\mathbf{j}}\right)$ - admissible damage frequency of decisive* members, which makes it possible to meet the recommended reliability class of a complex structure. In a statically determinate structure, $\mathbf{n}$ number of decisive members is found. In order to meet the reliability level $\mathbf{p}\left(\mathbf{t}_{\mathbf{3}}=\mathbf{4 . 3}\right)$ of a complex structure, it is necessary to reduce the damage frequency $\mathbf{q}\left(\mathbf{t}_{\mathbf{i}}\right)$ of decisive* members to that determined from the equation (3.2):

$$
\mathbf{q}\left(\mathbf{t}_{\mathbf{i}}\right)=<\mathbf{q}\left(\mathbf{t}_{\mathbf{3}}\right) / \mathrm{n}
$$

Coefficient $\mathbf{s}$ that recommends an increase of the load bearing capacity of decisive* members of the structure should be calculated from the formula (2.8): $\mathbf{s}=\left[\mathbf{1}-\mathbf{t}_{\mathbf{2}} \mathbf{v}_{\mathbf{i}}\right]$ / $\left[\mathbf{1}-\mathbf{t}_{\mathbf{i}} \mathbf{v}_{\mathbf{i}}\right]$. Table 3 shows the estimates of $\mathbf{s}$ coefficients computed from a detailed formula (3.3):

$$
s=\left[1-3.8 v_{i}\right] /\left[1-5.64926 v_{i}\right]
$$

In order to draw graphs of coefficients $\mathbf{s}$ which correct the coefficients of the load bearing capacity of decisive members estimated for RC2 class $(3,8)$, so that they would be applicable to RC 3 class complex structures $\left(t_{3}=4.3\right)$, coordinates $\lg _{10}(n=1000)$, which control the construction of nomographs in accordance with Example 3, were selected. 
Example 3. Statically determinate high-profile RC3 $\left(\mathbf{t}_{\mathbf{3}}=\mathbf{4 . 3}\right)$ class truss structure is given. It is composed of $n=1000$ decisive members that have the coefficient of variation of the load bearing capacity $\mathbf{v}_{\mathbf{1}}=\mathbf{0 . 0 6} ; 0.08 ; 0.09 ; 0.1$. The recommended structure reliability is: $\mathbf{p}\left(\mathbf{t}_{\mathbf{3}}=\mathbf{4 . 3}\right)=\mathbf{0 . 9 9 9 9 9 1 4 6 0 0 9 4 5 2 4}$, and the admissible damage frequency of the structure is: $\mathbf{q}\left(\mathbf{t}_{3}\right)=\mathbf{0 . 0 0 0 0 0 8 5 3 9 9 0 5 4 7 6}$. It is necessary to estimate coefficients $\mathbf{s}$ which increase the load bearing capacity of decisive members of high-profile RC3 class structure composed of $n=1000$ members dimensioned in accordance with RC2 class [9]. The admissible damage frequency of the members is: $q_{i}(t)=q\left(t_{3}\right):(n=1000)=0.000000008539906$, the recommended reliability of the members: $\mathbf{p}_{\mathbf{i}}(\mathbf{t})=\mathbf{1}-\mathbf{q}_{\mathbf{i}}(\mathbf{t})=\mathbf{0 . 9 9 9 9 9 9 9 9 1 4 6 0 0 9 4}$. The reliability index $t$ of the decisive members amounts to: $\mathbf{t}=\mathbf{5 . 6 3 9 2 6}$. Table 3 shows estimates of coefficients $\mathbf{s}$ from the detailed formula: $s=\left[\mathbf{1}-3.8 v_{i}\right] /\left[\mathbf{1}-5.63926 v_{i}\right]$.

Table 3

Coefficients $\mathbf{s}$ for $\mathrm{n}=1000$, controlling the graphs of RC3 structure nomograph (Fig. 3)

\begin{tabular}{|c|l|}
\hline $\mathbf{v}_{\mathbf{1}}=\mathbf{0 . 0 6}$ & $\mathbf{s}=(1-3.8 \times 0.06) /(1-5.63926 \times 0.06)=0.772 / 0.66164=\mathbf{1 . 1 6 6 8}$ \\
\hline $\mathbf{v}_{\mathbf{1}}=\mathbf{0 . 0 8}$ & $\mathbf{s}=(1-3.8 \times 0.08) /(1-5.63926 \times 0.08)=0.696 / 0.54886=\mathbf{1 . 2 6 8 1}$ \\
\hline $\mathbf{v}=\mathbf{0 . 0 9}$ & $\mathbf{s}=(1-3.8 \times 0,09) /(1-5.63926 \times 0.09)=0.658 / 0.49247=\mathbf{1 . 3 3 6 1}$ \\
\hline $\mathbf{v}_{\mathbf{1}}=\mathbf{0 . 1 0}$ & $\mathbf{s}=(1-3.8 \times 0.10) /(1-5.63926 \times 0.10)=0.620 / 0.43607=\mathbf{1 . 4 2 1 8}$ \\
\hline
\end{tabular}

Table 4 presents coefficients $\mathbf{s}$ estimated with the interpolation of those given in Table 3 for $\mathrm{n}=1000$. They are used to compile nomographs shown in Fig. 3. Table 4 can be applied to the direct estimation of coefficients $\mathbf{s}$ for coefficients of variation $\mathbf{v}=0.06$; $0.08 ; 0.09 ; 0.1$ of the load bearing capacity of members in a RC3 class complex structure with the reliability index $\mathbf{t}_{\mathbf{3}} \mathbf{=} \mathbf{4 . 3}$.

Table 4

Coefficients s for increasing the load bearing capacity of decisive members of RC 3 class structure

\begin{tabular}{|c|c|c|c|c|c|c|}
\hline $\mathrm{n}$ & 10 & 50 & 100 & 200 & 500 & 1000 \\
\hline $\mathrm{v}=0.06$ & $\mathbf{1 . 0 5 5 6}$ & $\mathbf{1 . 0 9 4 5}$ & $\mathbf{1 . 1 1 1 2}$ & $\mathbf{1 . 1 2 7 9}$ & $\mathbf{1 . 1 5 0 1}$ & $\mathbf{1 . 1 6 6 8}$ \\
\hline $\mathrm{v}=0.08$ & $\mathbf{1 . 0 8 9 4}$ & $\mathbf{1 . 1 5 1 8}$ & $\mathbf{1 . 1 7 8 7}$ & $\mathbf{1 . 2 0 5 6}$ & $\mathbf{1 . 2 4 1 2}$ & $\mathbf{1 . 2 6 8 1}$ \\
\hline $\mathrm{v}=0.09$ & $\mathbf{1 . 1 1 2 0}$ & $\mathbf{1 . 1 9 0 4}$ & $\mathbf{1 . 2 2 4 1}$ & $\mathbf{1 . 2 5 7 8}$ & $\mathbf{1 . 3 0 2 4}$ & $\mathbf{1 . 3 3 6 1}$ \\
\hline $\mathrm{v}=0.10$ & $\mathbf{1 . 1 4 0 6}$ & $\mathbf{1 . 2 3 8 9}$ & $\mathbf{1 . 2 8 1 2}$ & $\mathbf{1 . 3 2 3 5}$ & $\mathbf{1 . 3 7 9 5}$ & $\mathbf{1 . 4 2 1 8}$ \\
\hline $\mathrm{x}=\lg _{10}(\mathrm{n})$ & 1 & 1.699 & 2 & 2.301 & 2.699 & 3 \\
\hline
\end{tabular}

Fig. 3 shows linear graphs of coefficients $\mathbf{s}$ constructed for RC 3 structure with the index $t_{3}=4.3$ and $v=0.06 ; 0.08 ; 0.09 ; 0.1$. $\mathrm{s}$ coefficients can be interpolated and extrapolated directly from the graphs in Fig. 5. The case of $v=0.12$ was omitted in Table 4 
and in Fig. 5 because in high-profile structures, heightened quality control should be imposed. In order to facilitate interpolation and extrapolation of coefficients $\mathbf{s}$, Table 3 provides the data for the logarithmic scale refinement, thus extending the determination of $\mathbf{s}$ coefficients.

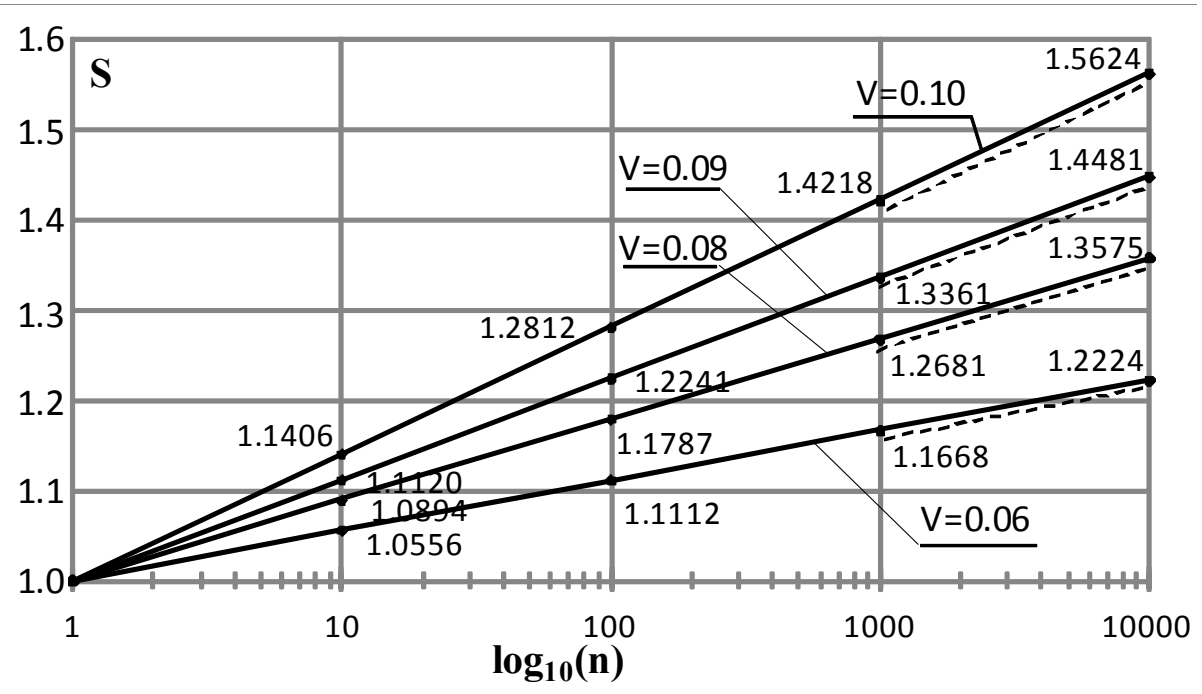

Fig. 3. coefficients $\mathbf{s}$ for increasing the load bearing capacity of decisive* members of RC3 class structure $\left(t_{\mathbf{3}}=\mathbf{4 . 3}\right)$.

\section{SUMMARY}

Failures and collapses of large complex steel structures indicate that, while estimating structure reliability, it is common to disregard the impact of a number of decisive elements (with joints included) on structural safety. The estimation of the reliability of complex structures with conventional methods in accordance with the code is prone to systematic errors. The latter depend on geometric solutions employed in complex structures, and also on number of decisive members and their connections. Errors result from unacceptable mathematical operations on quantiles, and also from frequent underestimation of the load bearing capacity of joints. Quantiles are all characteristic and computational physical quantities of a structure, which are given in the code. The paper presents the method of estimating the impact of a number of decisive members on the load bearing capacity and reliability of statically determinate structures. For the method to be applied, it is sufficient to: 1) estimate the coefficients of variation of the load bearing capacity of decisive members, 2) dimension the structure members in accordance with the binding code, 3 ) estimate $\mathbf{s}$ coefficients which indicate the need for increasing the load bearing capacity of decisive members above the estimations 
that result from the code. That constitutes a major advantage of the method presented in the paper. It is widely believed that the coefficient of variation $v_{i}$ of the load bearing capacity of well fabricated decisive members approximately ranges $\mathrm{v}=0.06-0.08$, and the reliability of decisive members in metal structures dimensioned in accordance with the code meets RC2 class requirements. The joints of decisive members, however, are commonly underestimated, both when designed and executed, hence a need arises to consider joints as decisive members. The reliability of statically loaded complex structures is naturally facilitated by fabrication of precast units in steps, which results in adopting larger sections than theoretically necessary. A greater number of independent sets of static loads is also advantageous for heightening the safety of a structure. If the method were consistently applied, that would make it possible to avoid more than $70 \%$ of failures and collapses of statically determinate complex structures, or of statically determinate segments of large structural systems. The remaining $30 \%$ of failures are attributed to gross errors. The calculations for bars integrated with joints in truss nodes are sensitive to variation coefficients $\mathbf{v}_{\mathbf{i}}$ of the load bearing capacity of joints. Therefore, the load bearing capacity of joints, especially those welded in assembly, should be higher than the load bearing capacity of bars, in accordance with, e.g. work [7]. Graphs of coefficient $\mathbf{s}$ in Figs 2 and 3 indicate considerable influence of the design quality, workmanship and assembly on the structure reliability shown in variation coefficients $\mathrm{v}_{\mathrm{i}}$.

General conclusion: Decisive members in statically determinate complex structures need to be dimensioned in such a manner so that the target reliability of the structure is attained. In the process, it is necessary to account for an increase in damage frequency as the number of decisive members in a structure increases. Also, in bar decisive members, decisive bar connections to nodes should be included, or the load bearing capacity of decisive bars integrated in series with joints ought to be estimated in accordance with work [7].

The load bearing capacity and reliability of statically indeterminate structures was discussed in other works [12], in which it was shown how it is possible to apply probabilistic optimisation of the load bearing capacity of complex structures without increasing the load bearing capacity of members above the requirements stated in construction codes. Also, attention should be paid to a significant role the structure geometry [5],[6]12] played in the optimisation of the complex structure load bearing capacity and reliability. Introducing, intentionally, the bars that couple decisive members into kinematically admissible failure mechanisms (KAFM) [12], reverses the tendency for reliability to decrease with a growing number of decisive members. Alternatively, that yields enhanced load bearing capacity and reliability of the structure [12]. The power of the latter tendency is manifested in regular bar truss structures $[5 ; 12]$ with triangular, square and rectangular mesh, including the structures where every other mesh aperture is empty. The structures that do not pose hazards shown in study [6], constructed from members dimensioned in accordance with the binding codes, have higher load bearing capacity and reliability than those recommended by the code [9]. 


\section{REFERENCES}

1. A. Biegus, Fundamentals of Probabilistic Analysis of Structure Reliability (in Polish). Publication of the Wrocław University of Technology 1996.

2. M. Gwóżdż, A. Machowsкi, Selected Problems and Building Structure Calculations with Probabilistic Methods (in Polish). Publication of the Cracow University of Technology. Cracow 2011.

3. B. Kopociński, Outline of Theory of Rehabilitation and Reliability (in Polish). PWN, Warsaw 1973.

4. Z. Kowal, On Causes of Decrease in Reliability of Hall Steel Structures (in Polish). Remo 2009. Wiadomości Konserwatorskie 26/2009, pp. 565-573.

5. Z. KowaL, The formation of space bar structures supported by the system reliability theory. Arch.pf Civil and Mechanical Engineering.Vol. XI. No. 1.2011. Polish Ac. of Sciences - Wrocław Branch., Wrocław Univ. of Technology.

6. Z. KowAl, Hazards associated with the load-bearing capacity of bar space structures during assembly and performance. Promysłowe budiwnyctwo ta inżenerni sporudy. Kiev 2011, No 3. pp. 34-40.

7. Z. Kowal, Dimensioning Bar Joints in Trusses from the Structure Reliability Standpoint (in Polish). Conference paper, Krynica 2010, pp. 675-682.

8. J. Murzewski, Reliability of Engineering Structures (in Polish). Arkady, Warsaw 1989.

9. Eurocode PN-EN - 1990:2002. Fundamentals of Structural Design

10. Eurocode PN-EN 1993. Design of Steel Structures.

11. R. Zielíśsi, Statistical Tables (in Polish). PWN.Warszawa 1972.

12. Z. Kowal, Probabilistic Optimisation of Bearing Capacity of Columns in Conventional Halls (in Polish). Conference paper, Krynica 2012. 